\title{
Epidemiology of Human Infections with Avian Influenza A(H7N9) Virus in China
}

\begin{abstract}
Qun Li, M.D.\#, Lei Zhou, M.D.", Minghao Zhou, Ph.D.\#, Zhiping Chen, M.D.\#, Furong Li, M.D.\#, Huanyu Wu, M.D., Nijuan Xiang, M.D., Enfu Chen, M.P.H., Fenyang Tang, M.D., Dayan Wang, M.D., Ling Meng, M.D., Zhiheng Hong, M.D., Wenxiao Tu, M.D., Yang Cao, M.D., Leilei Li, Ph.D., Fan Ding, M.D., Bo Liu, M.D., Mei Wang, M.D., Rongheng Xie, M.D., Rongbao Gao, M.D., Xiaodan Li, M.D., Tian Bai, M.D., Shumei Zou, M.D., Jun He, M.D., Jiayu Hu, M.D., Yangting Xu, M.D., Chengliang Chai, M.D., Shiwen Wang, M.D., Yongjun Gao, M.D., Lianmei Jin, M.D., Yanping Zhang, M.D., Huiming Luo, M.D., Hongjie Yu, M.D., M.P.H., Jianfeng He, M.D., Qi Li, M.D., Xianjun Wang, M.D., Lidong Gao, M.D., Xinghuo Pang, M.D., Guohua Liu, M.D., Yansheng Yan, M.D., Hui Yuan, M.D., Yuelong Shu, Ph.D., Weizhong Yang, M.D., Yu Wang, M.D., Fan Wu, M.D., Timothy M. Uyeki, M.D., M.P.H., M.P.P., Zijian Feng, M.D., M.P.H. The Public Health Emergency Center (Q.L., L.Z., N.X., L.M., Z.H., W.T., Y.C., L.L., F.D., B.L., M.W., Y.G., L.J., Y.Z., Z.F.) and National Institute for Viral Disease Control and Prevention (D.W., R.G., X.L., T.B., S.Z., S.W., Y.S.), Chinese Center for Disease Control and Prevention (H.L., H.Y., W.Y., Y.W.), Chinese Field Epidemiology Training Program (R.X.), and Beijing Municipal Center for Disease Control and Prevention (X.P.), Beijing, Jiangsu Provincial Center for Disease Control and Prevention (M.Z., F.T.) and Nanjing Prefecture Center for Disease Control and Prevention (Y.X.), Nanjing, Zhejiang Provincial Center for Disease Control and Prevention, Hangzhou (Z.C., E.C., C.C.), Anhui Provincial Center for Disease Control and Prevention, Hefei (F.L., Jun He), Shanghai Municipal Center for Disease Control and Prevention, Shanghai (H.W., J. Hu, F.W.), Guangdong Provincial Center for Disease Control and Prevention, Guangzhou (Jianfeng He), Hebei Provincial Center for Disease Control and Prevention, Shijiazhuang (Q.L.), Shandong Provincial Center for Disease Control and Prevention, Jinan (X.W.), Hunan Provincial Center for Disease Control and Prevention, Changsha (L.G.), Henan Provincial Center for Disease Control and Prevention, Zhengzhou (G.L.), Fujian Provincial Center for Disease Control and Prevention, Fuzhou (Y.Y.), and Jiangxi Provincial Center for Disease Control and Prevention, Nanchang (H.Y.) - all in China; and Influenza Division, Centers for Disease Control and Prevention, Atlanta (T.M.U.).

\# These authors contributed equally to this work.
\end{abstract}

\footnotetext{
Abstract

BACKGROUND—The first identified cases of avian influenza A(H7N9) virus infection in humans occurred in China during February and March 2013. We analyzed data obtained from field

Address reprint requests to Dr. Feng at the Public Health Emergency Center, Chinese Center for Disease Control and Prevention, Beijing, 102206, China, or at fengzj@ chinacdc.cn; or to Dr. Fan Wu at Shanghai Municipal Center for Disease Control and Prevention, 1380 Zhongshan W. Rd., Shanghai, 200336, China, or at fwu@ scdc.sh.cn.

The views expressed in this article are those of the authors and do not represent the official policy of the Chinese Center for Disease Control and Prevention or the U.S. Centers for Disease Control and Prevention.

Disclosure forms provided by the authors are available with the full text of this article at NEJM.org.
} 
investigations to describe the epidemiologic characteristics of H7N9 cases in China identified as of December 1, 2013.

METHODS—Field investigations were conducted for each confirmed case of H7N9 virus infection. A patient was considered to have a confirmed case if the presence of the H7N9 virus was verified by means of real-time reverse-transcriptase-polymerase-chain-reaction assay (RTPCR), viral isolation, or serologic testing. Information on demographic characteristics, exposure history, and illness timelines was obtained from patients with confirmed cases. Close contacts were monitored for 7 days for symptoms of illness. Throat swabs were obtained from contacts in whom symptoms developed and were tested for the presence of the H7N9 virus by means of realtime RT-PCR.

RESULTS-Among 139 persons with confirmed H7N9 virus infection, the median age was 61 years (range, 2 to 91 ), $71 \%$ were male, and $73 \%$ were urban residents. Confirmed cases occurred in 12 areas of China. Nine persons were poultry workers, and of 131 persons with available data, $82 \%$ had a history of exposure to live animals, including chickens (82\%). A total of 137 persons (99\%) were hospitalized, 125 (90\%) had pneumonia or respiratory failure, and 65 of 103 with available data $(63 \%)$ were admitted to an intensive care unit. A total of 47 persons (34\%) died in the hospital after a median duration of illness of 21 days, 88 were discharged from the hospital, and 2 remain hospitalized in critical condition; 2 patients were not admitted to a hospital. In four family clusters, human-to-human transmission of H7N9 virus could not be ruled out. Excluding secondary cases in clusters, 2675 close contacts of case patients completed the monitoring period; respiratory symptoms developed in 28 of them (1\%); all tested negative for H7N9 virus.

CONCLUSIONS-Most persons with confirmed H7N9 virus infection had severe lower respiratory tract illness, were epidemiologically unrelated, and had a history of recent exposure to poultry. However, limited, nonsustained human-to-human H7N9 virus transmission could not be ruled out in four families.

The first identified cases of human infection with a novel influenza A(H7N9) virus occurred in eastern China during February and March 2013 and were characterized by rapidly progressive pneumonia, respiratory failure, the acute respiratory distress syndrome (ARDS), and fatal outcomes. ${ }^{1}$ We analyzed available data from field investigations to characterize the descriptive epidemiology of laboratory-confirmed cases of avian influenza A(H7N9) virus infection in humans that were reported to the Chinese Center for Disease Control and Prevention (China CDC) as of December 1, 2013. In this report, we summarize the epidemiologic findings of case investigations and follow-up monitoring of close contacts of persons with confirmed cases of H7N9 virus infection who have been identified to date. Investigations are ongoing.

\section{METHODS CASE DEFINITIONS}

The case definitions of suspected and confirmed human infection with H7N9 virus were based on the H5N1 case definitions, as recommended by the World Health Organization (WHO) in 2006 (Section S1 in the Supplementary Appendix, available with the full text of this article at NEJM.org). ${ }^{2}$ A patient was considered to have a confirmed case if the presence 
of the H7N9 virus was verified by means of real-time reverse-transcriptase-polymerasechain-reaction assay (RT-PCR), viral isolation, or serologic testing. The laboratory test assays for H7N9 virus that we performed have been described previously (Section S2 in the Supplementary Appendix). ${ }^{1}$

\section{IDENTIFICATION OF CASES}

Suspected cases of H7N9 virus infection among hospitalized patients with pneumonia were identified through the Chinese surveillance system for pneumonia of unknown origin, which was established in 2004. ${ }^{3,4}$ Beginning on April 3, 2013, enhanced surveillance was implemented for suspected cases of H7N9 virus infection among persons with mild or moderate illness. ${ }^{5}$ Persons with suspected cases of H7N9 virus infection with mild or moderate illness were identified from the Chinese sentinel surveillance system for influenzalike illness, which has been described previously. ${ }^{6}$ Once each suspected case of H7N9 virus infection was identified, the local CDCs, including prefecture and provincial CDCs, conducted the initial field investigations and obtained respiratory specimens, which were shipped to the National Influenza Center of the China CDC in Beijing for H7N9 laboratory testing. The China CDC was involved in field investigations of the first 82 confirmed cases of H7N9 virus infection; local CDCs conducted field investigations of subsequent confirmed cases.

\section{DATA COLLECTION}

The National Health and Family Planning Commission (NHFPC) determined that the collection of data from H7N9 case patients and their close contacts was part of a continuing public health investigation of an outbreak and was exempt from institutional review board assessment. Data were collected through a review of medical records and interviews with relatives, contacts, and health care workers who provided medical care for the case patients. We collected information on the dates of illness onset, visits to clinical facilities, hospitalization, and clinical outcomes. Epidemiologic data were collected through interviews and field observations and were reported to the China CDC. Investigators interviewed the relatives of each patient with a confirmed case of H7N9 virus infection to determine exposure histories during the 2 weeks before the onset of the illness, including the dates, times, frequency, and patterns of exposures to poultry or other animals such as swine and wild birds. All epidemiologic information that was collected during the field investigations, including exposure history, timelines of events, and identification of close contacts, was cross-validated, since we were unable to interview any critically ill H7N9 case patients. Households and places known to have been visited by the case patients in the 2 weeks before the onset of illness were investigated to assess exposures to poultry and swine, as well as environmental exposures. We also collected information on control measures implemented by national, provincial, and municipal governments.

\section{IDENTIFICATION AND FOLLOW-UP OF CLOSE CONTACTS}

We defined close contacts of patients with confirmed H7N9 virus infection as described previously for H5N1 field investigations ${ }^{7}$ (Section S3 in the Supplementary Appendix); once we identified the close contacts, we monitored them daily for 7 days for symptoms of illness 
and collected throat swabs from contacts in whom symptoms developed to test for the presence of H7N9 virus.

Antiviral chemoprophylaxis was not provided to close contacts. Paired serum samples were obtained for H7N9 serologic testing from patients with suspected H7N9 virus infection who did not have respiratory specimens available. Oseltamivir treatment was recommended for close contacts in whom symptoms developed (Section 3 in the Supplementary Appendix). Data on demographic characteristics and exposure were collected for close contacts.

\section{STATISTICAL ANALYSIS}

We used descriptive statistics to summarize the epidemiologic characteristics and H7N9 testing results for persons with suspected cases of H7N9 virus infection, for those with confirmed cases, and for close contacts of those with confirmed cases. The methods we used for estimating the incubation period have been described previously. ${ }^{8}$

\section{RESULTS EPIDEMIOLOGIC CHARACTERISTICS OF CONFIRMED CASES}

From March 25 through December 1, 2013, respiratory specimens from 1372 hospitalized patients with pneumonia of unexplained origin were tested, and 104 patients (7.6\%) were confirmed to be infected with the H7N9 virus. Of 2,130,049 respiratory specimens obtained from outpatients with an influenza-Iike illness through the sentinel surveillance system for influenza-like illness, $8(0.0004 \%)$ tested positive for the H7N9 virus. As of December 1, 2013, a total of 139 laboratory-confirmed cases of H7N9 virus infection (Fig. 1 and 2) and 1 suspected case had been identified; cases were identified in the following 10 provinces and 2 municipalitits: Zhejiang (50 confirmed cases), Shanghai (33 confirmed cases), Anhui (4 confirmed cases), Henan (4 confirmed cases), Hunan( 2 confirmed cases, Beijing (2 confirmed cases)), Shandong ( 2 confirmed cases), Guangdong (2confirmed cases), and Hebei (1 confirmed case).

The median age of patients with confirmed H7N9 virus infection was 61 years (range, 2 to 91; interquartile range, 46 to 73 ); 58 cases (42\%) occurred in persons 65 years of age of older, and $4(3 \%)$ were in children younger than 5 years of age, all of whom had clinically mild upper respiratory illness (see Fig. S1 in the Supplementary Appendix for the age distribution). Most confirmed cases (71\%) occurred in males, $73 \%$ of the case patients were urban residents, and 79 of 108 patients with available data (73\%) had underlying medical conditions (TabIe 1). Among 70 of 79 case patients with sufficient data for a more specific classification of underlying conditions, $54(77 \%)$ were considered to be at increased risk for influenza complications owing to age ( $<5$ years or 265 years) or prevalence of certain underlying medical conditions. ${ }^{9}$ Nine of the patients with confirmed cases $(6 \%)$ worked as poultry workers: 6 slaughtered poultry at a live poultry market, 2 transported live poultry, and 1 raised and traded pigeons.

A total of 137 of 139 patients with confirmed H7N9 virus infection (99\%) were hospitalized (Table 2), and 125 (90\%) had pneumonia or respiratory failure. Among the 103 patients with confirmed cases for whom data were available, isolation precautions were instituted for 65 
(63\%) in an intensive care unit (ICU) because of severe lower respiratory tract disease.

Among patients with cases identified as of December 1, a total of 47 with confirmed H7N9 virus infection (34\%) and 1 with suspected infection had died of the acute respiratory distress syndrome (ARDS) or multiorgan failure in the hospital, 2 critically ill patients with confirmed cases remained hospitalized, and 88 had been discharged. One 2-year-old boy and one 25-year-oId woman with mild upper respiratory tract illness were not hospitalized. Among 139 confirmed cases of H7N9 virus infection, 49 (35\%) were confirmed by means of virus isolation, 1 by means of serologic testing, and the remaining 89 (64\%) by means of nucleic acid detection. Five of 139 persons with virologically confirmed cases also tested seropositive for H7N9 virus antibodies with the use of a turkey red-cell hemagglutinin inhibition assay (Table 1).

Data on recent exposure to animals were available for 131 of the 139 patients with confirmed H7N9 virus infection. Of these, 107 (82\%) reported a history of recent exposure to animals (Table 1): $88(82 \%)$ to chickens, 24 (22\%) to ducks, and $6(6 \%)$ to swine; the exposures occurred either while they were working at or while they were visiting a live animal market. Other animals that these 107 patients reported having been exposed to included pigeons, geese, quail, wild birds, pet birds, cats, and dogs. Information on a history of exposure to live animals is unclear for 8 patients with confirmed H7N9 virus infection, either because the investigations are still ongoing or be-cause data on exposure could not be obtained from patients owing to critical illness resulting in death. The estimated median incubation period in 23 patients with confirmed cases for whom detailed data on animal and environmental exposures were available was 6 days (range, 1 to 10)(Table S1 in the Supplementary Appendix).

\section{FAMILY CLUSTERS}

As of December 1, four family clusters had been identified in three areas. Detailed information on exposure, date of illness onset, and follow-up for these family clusters is provided in Figure 3, and in Section S4 in the Supplementary Appendix. The first family cluster comprised two persons with confirmed H7N9 virus infection and one with a suspected infection. The index case patient and his father (confirmed case 2) lived in the same house, and the index patient's brother (confirmed case 1) lived with his wife nearby. After the index case patient (suspected case 1) became ill, his brother (confirmed case 1) and his father (confirmed case 2) had prolonged, close, unprotected contact with him, including eating together, providing care, and accompanying him to seek medical care before his hospitalization (Fig. 3A, and Section S4 in the Supplementary Appendix). None of the three members of the cluster raised poultry or other animals, none brought live poultry into their home, and none had direct contact with sick or dead poultry. The index case patient had visited a live poultry market, purchased a chicken, observed the slaughtering process, brought the freshly killed chicken home, and prepared, cooked, and ate the chicken within 2 weeks before the onset of his illness.

The second family cluster included two persons with confirmed H7N9 virus infection (Fig. 3B, and Section S4 in the Supplementary Appendix). After the father (confirmed case 1) became ill, his daughter (confirmed case 2 ) had prolonged, close, unprotected contact with 
him, including eating together, providing care, and accompanying him to seek medical care before his hospital admission. She also provided unprotected bedside hospital care for her father during the period from March 11 through March 15. Diarrhea developed in the father, and the daughter washed her father's diarrhea-soiled underwear on March 18 while wearing gloves. The father had visited a live poultry market 7 days before the onset of his illness. The daughter did not raise poultry or animals at home and had not had any exposures to animals (i.e., had not brought live poultry into the home or visited a live poultry market or had any direct or indirect contact with poultry or pigs).

The third family cluster included a wife and husband with confirmed H7N9 virus infection (Fig. 3C, and Section S4 in the Supplementary Appendix). After the wife (confirmed case 1) became ill, her husband (confirmed case 2) had prolonged, close, unprotected contact with her, including eating with her, providing care for her, and accompanying her to seek medical care before her admission to the hospital. He also provided unprotected bedside care for his wife in the hospital on April 2 and April 3. The wife had visited a live poultry market within 7 days before the onset of her ill-ness. The husband did not raise poultry or animals at home and had not had any exposures to animals (i.e., had not brought live poultry into the home or visited a live poultry market or had any direct or indirect contact with poultry or pigs).

The fourth family cluster included a father and son with confirmed H7N9 virus infection (Fig. 3D, and Section S4 in the Supplementary Appendix). After the father (confirmed case 1) became ill, his son (confirmed case 2) had direct and very close contact with him, including hugging, sitting, eating, and sleeping together. The family did not raise poultry or other animals and did not bring live poultry into their home. Neither the father nor the son had any direct contact with poultry and neither had visited a live poultry market within 2 weeks before the onset of illness. H7N9 virus infection in the son was identified through monitoring and testing of 12 close contacts of confirmed case 1.

\section{MEDICAL CARE TIMELINES}

Among the 137 patients with confirmed H7N9 virus infection for whom data were available ( $99 \%$ of the 139 patients with confirmed cases), the median time from the onset of illness to the first medical visit was 1 day, and patients were hospitalized a median of 4 days after the onset of illness (Table 2). Among 109 of the patients with available data on oseltamivir administration, $79(72 \%)$ received oseltamivir treatment beginning a median of 6 days (range, 0 to 15) after the onset of illness. ARDS developed during the course of hospitalization in 48 of 83 patients with confirmed H7N9 virus infection for whom data were available (58\%) after a median of 7 days, and 47 patients died a median of 21 days after the onset of illness.

\section{CLOSE CONTACTS}

As of December 1, excluding data on patients with confirmed cases who were close contacts of index patients in the four family clusters, data were available for 2675 close contacts of the 139 patients with confirmed cases, in Zhejiang (1133 contacts), Jiangsu (552), Shanghai (378), Anhui (135), Fujian (111), Hunan (91), Guangdong (103), Beijing (43), Henan (42), Jiangxi (46), Hebei (30), and Shandong (11). Among the 1953 close contacts of the patients 
with confirmed cases for whom demographic information was available, $1039(53 \%)$ were health care workers, $515(26 \%)$ were family members or relatives, and $399(20 \%)$ were social contacts. As of December 1, all 2675 contacts had been followed for up for 7 days; among these close contacts, respiratory symptoms developed during the 7-day surveillance period in 28 (1\%): 9 household members, 1 medical intern, 1 patient who shared a room with a confirmed case patient, and 17 health care workers (Section S5 in the Supplementary Appendix). Throat swabs were collected from these 28 ill contacts a median of 1 day (range, 0 to 8 ) after the onset of illness. All 28 ill close contacts were negative for the H7N9 virus, as assessed by means of real-time RT-PCR.

\section{CONTROL MEASURES}

On April 3, 2013, the NHFPC established the Joint Prevention and Control Mechanism (JPCM) to lead the national response to H7N9, issued the first national H7N9 technical guideline, and implemented enhanced surveillance for H7N9 virus infection among persons with influenza-like illness in the existing sentinel surveillance system (Fig. 1). A local JPCM was established in four provinces - Shanghai, Jiangsu, Zhejiang, and Anhui — on April 7. The China CDC established an Internet-based national H7N9 case-reporting system for provinces and municipalities on April 19. The national H7N9 technical guideline was revised on May 10. Of the 10 affected provinces and 2 municipalities, all except Guangdong province launched emergency responses immediately after detection of a confirmed H7N9 case (Fig. S2 in the Supplementary Appendix) and closed live poultry markets for 1 to 2 months after an H7N9 case occurred. (Live poultry markets have been forbidden in Beijing since 2005.) Live poultry markets in Guangdong were monitored after an H7N9 case was identified, and daily poultry deliveries were limited to ensure that the unsold poultry that was kept overnight did not exceed $10 \%$ of the poultry delivered daily.

The outbreak can be characterized by five phases (Fig. 1, and Fig. S2 in the Supplementary Appendix): the initial phase from February 17 through March 27, with sporadic cases identified in Shanghai, Zhejiang, and Jiangsu provinces in eastern China; the epidemic peak from March 28 through April 18, during which 98 of the 139 total confirmed cases (71\%) were reported, in two municipalities and eight provinces; a declining phase from April 19 through May 31, with a sharp reduction in cases after closure of live poultry markets in one municipality and eight provinces; an interepidemic phase from June 1 through September 30 , with only 2 confirmed cases reported; and a reemergence phase beginning October 1 , with 5 new confirmed H7N9 cases as of December 1.

\section{DISCUSSION}

An outbreak of human infections with a novel avian influenza A(H7N9) virus began in February 2013 and as of December 1 had resulted in 139 confirmed cases identified in 10 provinces and 2 municipalities, primarily in eastern China. Although persons in a wide age spectrum were affected (age range, 2 to 91 years), the majority of patients with confirmed H7N9 virus infection were older (median age, 61 years), male (71\%), and urban residents (73\%), and most were considered to be at increased risk for complications from influenza owing to age ( $<5$ years or $\nsucceq 65$ years) or the prevalence of certain underlying medical 
conditions. Most patients were hospitalized with severe lower respiratory tract illness, ${ }^{10}$ with a case fatality proportion of $34 \%$ to date. Except for four case clusters among family members, most patients with confirmed H7N9 virus infection were epidemiologically unrelated. Two additional cases in critically ill patients were not included in our analyses; one patient had traveled from eastern China to Taiwan, where illness onset occurred and H7N9 virus infection was con-firmed, ${ }^{11}$ and one had traveled from southern China to the Hong Kong Special Administrative Region (HKSAR), where illness onset occurred and H7N9 virus infection was confirmed in early December. Since December 1, other confirmed cases of H7N9 virus infection have been identified in China, HKSAR, and Taiwan.

Human infections with avian influenza A (H7) viruses have been reported sporadically and are usually associated with exposures to poultry. ${ }^{12-14}$ Previous cases of $\mathrm{H} 7$ virus infection in humans have been characterized by mild illness (conjunctivitis or uncomplicated influenza) or by moderate illness (lower respiratory tract disease) that results in hospitalization. 12,13,15,16 Only one fatal case of $\mathrm{H} 7$ virus infection has been reported previously; that case occurred in an adult with a highly pathogenic avian influenza A (H7N7) virus infection. ${ }^{17}$ Many of the confirmed H7N9 case patients had critical and fatal illness, suggesting that the $\mathrm{H} 7 \mathrm{~N} 9$ virus is more virulent in humans than are other $\mathrm{H} 7$ viruses. The age distribution among H7N9 case patients is older and wider than that among H5N1 case patients in China (Fig. S1 in the Supplementary Appendix), whereas the case fatality proportion and the risk of death to date are lower than those for reported cases of H5N1 virus infection in China and worldwide. ${ }^{18,19}$

Early surveillance for H7N9 cases was focused on case finding for severe lower respiratory tract illness, and since April 3, expanded testing of outpatients with influenza-like illness has identified some mild cases of illness with H7N9 virus infection. ${ }^{20-22}$ Enhanced surveillance for less severe illness with H7N9 virus infection can help to determine the clinical spectrum of the illness and the total number of cases of H7N9 symptomatic illness and to inform an understanding of the true case fatality proportion. Since this H7N9 virus appears to have emerged recently to infect humans, population immunity is expected to be low, and persons of any age may be susceptible to infection. Owing to limitations of surveillance and testing, the number of patients with confirmed cases of H7N9 virus infection is likely to be an underestimate of all symptomatic cases that have occurred. One modeling study estimated that 27,000 cases of symptomatic H7N9 virus infection (95\% confidence interval, 9530 to 65,000 ) had occurred in China as of May $28 .^{23}$ Very limited data are available from small seroprevalence studies. ${ }^{24,25}$ Larger seroepidemiologic studies among different exposed groups can help define the true denominator of H7N9 virus infections.

The median time from the onset of illness to hospitalization among the 133 of 137 patients with confirmed H7N9 virus infection for whom data on hospitalization were available was 4 days, and the median time from the onset of illness to the development of ARDS among the 48 case patients with ARDS (of 83 patients for whom data on ARDS were available) was 7 days; the corresponding median times among patients with H5N1 virus infection were 7 days and 7.5 days. ${ }^{18,26}$ The median duration from the onset of illness to death among the 47 persons with confirmed cases who died was 21 days, which is much longer than the corresponding median of 11 days among 30 persons with fatal H5N1 cases in China. 
Patients with confirmed H7N9 infection received oseltamivir antiviral treatment a median of 6 days after the onset of illness (the median before April 3 was 9 days), probably owing to delays in seeking medical help and consequent delays in the identification of suspected influenza. Retrospective observational studies of influenza A(H1N1)pdm09 and H5N1 virus infections suggest that early oseltamivir treatment has the greatest clinical benefit but that starting treatment up to 5 days after the onset of illness may still reduce the risk of critical illness and death. ${ }^{27-30}$ Reports suggest that the H7N9 viruses isolated from humans and analyzed to date are resistant to adamantane antiviral agents and are susceptible to neuraminidase inhibitors. ${ }^{3,31,32}$ Early clinical suspicion of H7N9 virus infection and early administration of oseltamivir may help to reduce the severity of the disease. However, emergence of the R292K mutation in viral neuraminidase, which confers in vitro resistance to neuraminidase inhibitors, has been documented in H7N9 viruses during or after oseltamivir treatment in some critically ill patients. ${ }^{3,33}$ Therefore, to inform clinical management, clinical trials are needed to define efficacious therapy for $\mathrm{H} 7 \mathrm{~N} 9$ virus infection, including investigational agents and combination antiviral treatment.

Although the source of H7N9 virus infection in patients with confirmed cases who had exposure to animals cannot be verified without extensive H7N9 testing of animals, we suspect that it is likely to be infected poultry. H7N9 virus is a low pathogenic avian influenza A virus and does not cause identifiable illness or death in poultry; therefore, only laboratory testing can identify poultry infections. Among cases for which data are available, $82 \%$ occurred in patients who had exposure to live animals such as poultry or swine, including during visits to live animal markets. This raises the possibility of zoonotic H7N9 virus transmission from healthy-appearing poultry or swine to humans through direct or close contact or through exposure to environments that are contaminated with infected poultry or swine. For example, visiting a live poultry market, where avian influenza A viruses can be maintained and amplified, has been identified as a risk factor for $\mathrm{H} 5 \mathrm{~N} 1$ virus infection in Hong Kong ${ }^{34}$ and urban China. ${ }^{35,36}$ H7N9 virus has been isolated from poultry or environmental specimens collected at live poultry markets that were visited by some patients with confirmed cases before the onset of illness, and genetic sequencing results revealed the same H7N9 virus strain in viruses isolated from humans and from poultry or environmental samples from live poultry markets. ${ }^{37-40}$ Nine cases of H7N9 virus infection $(6.5 \%)$ occurred in poultry workers, including seven workers in live poultry markets.

Further evidence implicating live poultry markets in urban areas as the source of zoonotic H7N9 virus transmission is derived from case-control studies and the observed reduction in confirmed H7N9 cases after closure of live poultry markets. One case-control study identified direct contact with poultry or birds in the 2 weeks before the onset of illness, chronic medical conditions excluding hypertension, and exposure to an environment with poultry, including visiting a live poultry market, as risk factors associated with H7N9 virus infection. ${ }^{41} \mathrm{~A}$ larger study identified visiting live poultry markets as a risk factor, and contact with poultry as an independent risk factor, for H7N9 virus infection (unpublished data from the China CDC). One ecologic modeling study estimated that closure of live poultry markets reduced the mean daily number of H7N9 virus infections in the four most affected cities by 97 to $99 \% .42$ 
To date, follow-up prospective investigations of close contacts of patients with confirmed H7N9 virus infection have not conclusively established human-to-human H7N9 virus transmission. However, in four family clusters, limited, nonsustained human-to-human transmission of H7N9 virus after close, prolonged, unprotected contact with a symptomatic patient with H7N9 virus infection remains a possibility. Confirmed cases in three of the four clusters were identified in blood-related family members. Similar family clusters of H5N1 cases that occurred after common exposures to poultry or limited human-to-human transmission have been identified. $8,43,44$ Paired serum samples were obtained during the acute and convalescent stages of illness from contacts of case patients for further assessment of the potential for secondary human-to-human H7N9 virus transmission, including the potential identification of asymptomatic infections when testing is completed. Several studies have shown that H7N9 virus can bind to receptors in both the upper and lower airway in humans and can be transmitted variably from experimentally infected ferrets to susceptible ferrets by means of droplets. ${ }^{31,37,45,49}$ These studies support the observation that limited, nonsustained human-to-human transmission of H7N9 virus might occur.

Although the risk of human-to-human H7N9 virus transmission appears to be low, Chinese national guidelines recommend implementing control measures, such as prompt isolation of H7N9 patients; implementation of standard, contact, and droplet precautions by health care personnel in hospitals; and active monitoring of close contacts for illness. In addition, national guidelines recommend that antiviral treatment with oseltamivir should be administered as soon as possible in patients with suspected or confirmed cases of H7N9 virus infection.

Our study had several limitations. First, we were not able to collect detailed information from all patients on exposures, such as the times, frequency, intensity, and duration of exposures. Information on exposures is useful for estimating the incubation period after possible exposure to animals or live-animal markets and for evaluating risk factors for H7N9 virus infection. Second, we may not have identified all the close contacts of case patients. Third, we did not have a standard protocol and questionnaire to collect information from all contacts of the 139 patients with confirmed cases. However, the China CDC issued a guideline and protocol for field investigations of case patients and close contacts and since April 1 has provided training for personnel at all 31 provincial CDCs. A structured Internetbased reporting system was used to collect information on confirmed cases. This has helped to standardize data collection. Fourth, specimens were not available for H7N9 testing from some patients with suspected cases. It is also possible that by obtaining a single throat swab to test for the presence of the H7N9 virus, as compared with obtaining multiple specimens from different respiratory sites on different days, we could have missed detection of the virus in symptomatic contacts. Paired serum samples have not been obtained from some of the contacts because they have declined to have serum collected. Fifth, in-hospital mortality may be an underestimate of H7N9-associated mortality; one patient with a confirmed case was hospitalized, recovered, was discharged, and later died of an underlying medical condition but was not included as one of the 47 deaths among hospitalized patients with confirmed cases. Owing to surveillance limitations, some H7N9 virus infections have probably been missed ${ }^{23}$ Finally, since the outbreak is ongoing, we were unable to include in 
our analyses data from the patients with confirmed H7N9 cases that have been identified since December 1, 2013, or from their close contacts.

In summary, a novel avian influenza $\mathrm{A}(\mathrm{H} 7 \mathrm{~N} 9)$ virus has caused severe and fatal lower respiratory tract illness in persons in 12 different areas of China. Some clinically mild cases have been identified since the surveillance was widened, suggesting that there is a wide clinical spectrum of H7N9 virus infection. The epidemiologic findings suggest that most confirmed H7N9 cases were epidemiologically unrelated. On the basis of data from various studies to date, most case patients were probably exposed to H7N9 virus and infected during visits to live poultry markets, and the decline in cases during May followed the closure of live poultry markets in Shanghai municipality and nine provinces. Follow-up investigations of contacts of patients with confirmed H7N9 virus infection suggest that the risk of secondary transmission of H7N9 virus, including to health care personnel, is low at this time. However, in four family clusters, limited, nonsustained human-to-human transmission of H7N9 virus could not be ruled out and may have occurred.

On the basis of the experience with increased circulation of H5N1 virus among poultry during periods of cooler temperatures, the spread of H7N9 virus among poultry is likely to increase during the winter and spring months, with the potential for increased transmission to exposed persons. Therefore, enhanced surveillance for H7N9 virus among poultry and people, investigations of contacts of confirmed cases, and vi-rologic analyses to assess genetic changes that might suggest increased transmissibility among humans are all critical to informing prevention and control efforts and assessing the pandemic potential of this H7N9 virus. Prompt closure of live poultry markets is indicated in areas where new human cases of $\mathrm{H} 7 \mathrm{~N} 9$ virus infection are detected.

\section{Supplementary Material}

Refer to Web version on PubMed Central for supplementary material.

\section{Acknowledgments}

Supported by the China-U.S. Collaborative Program on Emerging and Re-emerging Infectious Diseases, a grant (201202006-01) from Research and Promotion of Key Technology on Health Emergency Preparation and Dispositions, a grant (No. KJYJ-2013-01-02) from the National Ministry of Science and Technology Emergency Research Project on human infection with avian influenza H7N9 virus, a grant (No. ZX201109) from Jiangsu Province Health Development Project with Science and Education, and a grant (No. 2009GG10002054) from Science and Technology Development Plan of Shandong Province.

We thank staff members of the Bureau of Disease Control and Prevention and Health Emergency Response Office of the National Health and Family Planning Commission and provincial and local departments of health for providing assistance with administration and data collection; staff members at county, prefecture and provincial government offices, CDCs, and hospitals in Shanghai and Beijing municipalities and Zhejiang, J iangsu, Anhui, Henan, Hunan, Shangdong, Fujian, Jiangxi, Hebei, and Guangdong provinces for providing assistance with field investigation administration and data collection; Jeffrey McFarland and Dale Hu from the U.S. CDC for comments on the manuscript; and Liangliang Cui from the Jinan Prefecture CDC and Jian Zhao from the Chinese CDC for assistance with the preparation of figures.

\section{REFERENCES}

1. Gao R, Cao B, Hu Y, et al. Human infection with a novel avian-origin influenza A (H7N9) virus. N Engl J Med 2013;368: 1888-97. [PubMed: 23577628] 
2. World Health Organization. WHO case definitions for human infections with influenza A (H5N1) virus (http://www.who.int/influenza/resources/documents/case_definition2006_08_29/en).

3. Yu H, Shu Y, Hu S, et al. The first confirmed human case of avian influenza A (H5N1) in Mainland China. Lancet 2006; 367:84. [PubMed: 16399159]

4. Xiang N, Havers F, Chen T, et al. Use of national pneumonia surveillance to describe influenza A(H7N9) virus epidemiology, China, 2004-2013. Emerg Infect Dis 2013;19:1784-90. [PubMed: 24206646]

5. National Health and Family Planning Commission, People's Republic of China. Guidelines of case clinical intervention and disease control and prevention of human infection with avian influenza H7N9 virus (in Chinese) (http://www.moh.gov.cn/mohwsyjbgs/fkzs/list.shtml).

6. Yu H, Cauchemez S, Donnelly CA, et al. Transmission dynamics, border entry screening, and school holidays during the 2009 influenza A (H1N1) pandemic, China. Emerg Infect Dis 2012;18:758-66. [PubMed: 22515989]

7. Wang H, Feng ZJ, Shu YL, et al. Probable limited person-to-person transmission of highly pathogenic avian influenza A (H5N1) virus in China. Lancet 2008; 371:1427-34. [PubMed: 18400288]

8. Huai Y, Xiang N, Zhou L, et al. Incubation period for human cases of avian influenza A (H5N1) infection, China. Emerg Infect Dis 2008;14:1819-21. [PubMed: 18976586]

9. Prevention and control of influenza with vaccines: recommendations of the Advisory Committee on Immunization Practices (ACIP), 2010. MMWR Recomm Rep 2010;59(RR-8):1-62. [Errata, MMWR Recomm Rep 2010;59:993, 1147.]

10. Gao HN, Lu HZ, Cao B, et al. Clinical findings in 111 cases of influenza A (H7N9) virus infection. N Engl J Med 2013;368:2277-85. [Erratum, N Engl J Med 2013;369:1869.] [PubMed: 23697469]

11. Chang SY, Lin PH, Tsai JC, Hung CC, Chang SC. The first case of H7N9 influenza in Taiwan. Lancet 2013;381:1621.

12. Koopmans M, Wilbrink B, Conyn M, et al. Transmission of H7N7 avian influenza A virus to human beings during a large outbreak in commercial poultry farms in the Netherlands. Lancet 2004; 363:587-93. [PubMed: 14987882]

13. Tweed SA, Skowronski DM, David ST, et al. Human illness from avian influenza H7N3, British Columbia. Emerg Infect Dis 2004;10:2196-9. [PubMed: 15663860]

14. Belser JA, Bridges CB, Katz JM, Tumpey TM. Past, present, and possible future human infection with influenza virus A subtype H7. Emerg Infect Dis 2009;15:859-65. [PubMed: 19523282]

15. Ostrowsky B, Huang A, Terry W, et al. Low pathogenic avian influenza A (H7N2) virus infection in immunocompromised adult, New York, USA, 2003. Emerg Infect Dis 2012;18:1128-31. [PubMed: 22710273]

16. Avian influenza A/(H7N2) outbreak in the United Kingdom. Euro Surveill 2007; 12(5):E070531.2.

17. Fouchier RA, Schneeberger PM, Rozendaal FW, et al. Avian influenza A virus (H7N7) associated with human conjunctivitis and a fatal case of acute respiratory distress syndrome. Proc Natl Acad Sci U S A 2004;101:1356-61. [PubMed: 14745020]

18. Cowling BJ, Jin L, Lau EH, et al. Comparative epidemiology of human infections with avian influenza A H7N9 and H5N1 viruses in China: a population-based study of laboratory-confirmed cases. Lancet 2013;382:129-37. [PubMed: 23803488]

19. World Health Organization. Cumulative number of confirmed human cases for avian influenza A(H5N1) reported to WHO, 2003-2013 (http://www.who.int/influenza/human_animal_interface/ EN_GIP_20131008CumulativeNumberH5N1cases.pdf).

20. Xu C, Havers F, Wang L, et al. Monitoring avian influenza A(H7N9) virus through national influenza-like illness surveillance, China. Emerg Infect Dis 2013;19:1289-92. [PubMed: 23879887]

21. Ip DK, Liao Q, Wu P, et al. Detection of mild to moderate influenza A/H7N9 infection by China's national sentinel surveillance system for influenza-like illness: case series. BMJ 2013;346:f3693. [PubMed: 23798720]

22. Lv H, Han J, Zhang P, et al. Mild illness in avian influenza A(H7N9) virus-infected poultry worker, Huzhou, China, April 2013. Emerg Infect Dis 2013;19: 1885-8. [PubMed: 24209963] 
23. Yu H, Cowling BJ, Feng L, et al. Human infection with avian influenza A H7N9 virus: an assessment of clinical severity. Lancet 2013;382:138-45. [PubMed: 23803487]

24. Bai T, Zhou J, Shu Y. Serologic study for influenza A (H7N9) among high-risk groups in China. N Engl J Med 2013; 368:2339-40. [PubMed: 23718151]

25. Xu W, Lu L, Shen B, Li J, Xu J, Jiang S. Serological investigations of subclinical influenza A(H7N9) infection among healthcare and non-healthcare workers in Zhejiang Province, China. Clin Infect Dis 2013;57:919-21. [PubMed: 23759348]

26. Yu H, Gao Z, Feng Z, et al. Clinical characteristics of 26 human cases of highly pathogenic avian influenza A (H5N1) virus infection in China. PLoS One 2008; 3(8):e2985. [PubMed: 18716658]

27. Yu H, Feng Z, Uyeki TM, et al. Risk factors for severe illness with 2009 pandemic influenza A (H1N1) virus infection in China. Clin Infect Dis 2011;52:457-65. [PubMed: 21220768]

28. Muthuri SG, Myles PR, Venkatesan S, Leonardi-Bee J, Nguyen-Van-Tam JS. Impact of neuraminidase inhibitor treatment on outcomes of public health importance during the 2009-2010 influenza $\mathrm{A}(\mathrm{H} 1 \mathrm{~N} 1)$ pandemic: a systematic review and meta-analysis in hospitalized patients. J Infect Dis 2013;207:553-63. [PubMed: 23204175]

29. Louie JK, Yang S, Acosta M, et al. Treatment with neuraminidase inhibitors for critically ill patients with influenza A (H1N1)pdm09. Clin Infect Dis 2012;55: 1198-204. [PubMed: 22843781]

30. Adisasmito W, Chan PK, Lee N, et al. Effectiveness of antiviral treatment in human influenza A(H5N1) infections: analysis of a Global Patient Registry. J Infect Dis 2010;202:1154-60. [PubMed: 20831384]

31. Zhou J, Wang D, Gao R, et al. Biological features of novel avian influenza A (H7N9) virus. Nature 2013;499:500-3. [PubMed: 23823727]

32. Watanabe T, Kiso M, Fukuyama S, et al. Characterization of H7N9 influenza A viruses isolated from humans. Nature 2013;501:551-5. [PubMed: 23842494]

33. Hu Y, Lu S, Song Z, et al. Association between adverse clinical outcome in human disease caused by novel influenza A H7N9 virus and sustained viral shedding and emergence of antiviral resistance. Lancet 2013;381:2273-9. [PubMed: 23726392]

34. Mounts AW, Kwong H, Izurieta HS, et al. Case-control study of risk factors for avian influenza A (H5N1) disease, Hong Kong, 1997. J Infect Dis 1999;180:505-8. [PubMed: 10395870]

35. Yu H, Feng Z, Zhang X, et al. Human influenza A (H5N1) cases, urban areas of People's Republic of China, 2005-2006. Emerg Infect Dis 2007;13:1061-4. [PubMed: 18214180]

36. Zhou L, Liao QH, Dong LB, et al. Risk factors for human illness with avian influenza A (H5N1) virus infection in China. J Infect Dis 2009;199:1726-34. [PubMed: 19416076]

37. Chen Y, Liang W, Yang S, et al. Human infections with the emerging avian influenza A H7N9 virus from wet market poultry: clinical analysis and characterisation of viral genome. Lancet 2013;381:1916-25. [PubMed: 23623390]

38. Wang C, Wang J, Su W, et al. Relationship between domestic and wild birds in live poultry market and a novel human H7N9 virus in China. J Infect Dis 2014; 209:34-7. [PubMed: 23990569]

39. Bao CJ, Cui LB, Zhou MH, Hong L, Gao GF, Wang H. Live-animal markets and influenza A (H7N9) virus infection. N Engl J Med 2013;368:2337-9. [PubMed: 23697471]

40. Han J, Jin M, Zhang P et al. Epidemiological link between exposure to poultry and all influenza A(H7N9) confirmed cases in Huzhou city, China, March to May 2013. Euro Surveill 2013;18(20): pii:20481.

41. Ai J, Huang Y, Xu K, et al. Case-control study of risk factors for human infection with influenza A(H7N9) virus in Jiangsu Province, China, 2013. Euro Surveill 2013;18:20510. [PubMed: 23827526]

42. Yu H, Wu JT, Cowling BJ et al. Effect of closure of live poultry markets on poultry-to-person transmission of avian influenza A H7N9 virus: an ecological study. Lancet 20131030 (Epub ahead of print).

43. Kandun IN, Wibisono H, Sedyaningsih ER, et al. Three Indonesian clusters of H5N1 virus infection in 2005. N Engl J Med 2006;355:2186-94. [PubMed: 17124016]

44. Ungchusak K, Auewarakul P, Dowell SF, et al. Probable person-to-person transmission of avian influenza A (H5N1). N Engl J Med 2005;352:333-40. [PubMed: 15668219] 
45. van Riel D, Leijten LM, de Graaf M, et al. Novel avian-origin influenza A (H7N9) virus attaches to epithelium in both upper and lower respiratory tract of humans. Am J Pathol 2013;183:1137-43. [PubMed: 24029490]

46. Belser JA, Gustin KM, Pearce MB, et al. Pathogenesis and transmission of avian influenza A (H7N9) virus in ferrets and mice. Nature 2013;501:556-9. [PubMed: 23842497]

47. Zhang Q, Shi J, Deng G, et al. H7N9 influenza viruses are transmissible in ferrets by respiratory droplet. Science 2013; 341:410-4. [PubMed: 23868922]

48. Xu L, Bao L, Deng W, et al. Novel avian-origin human influenza A (H7N9) can be transmitted between ferrets via respiratory droplets. J Infect Dis 2013924 (Epub ahead of print).

49. Richard M, Schrauwen EJ, de Graaf M, et al. Limited airborne transmission of H7N9 influenza A virus between ferrets. Nature 2013;501:560-3. [PubMed: 23925116] 


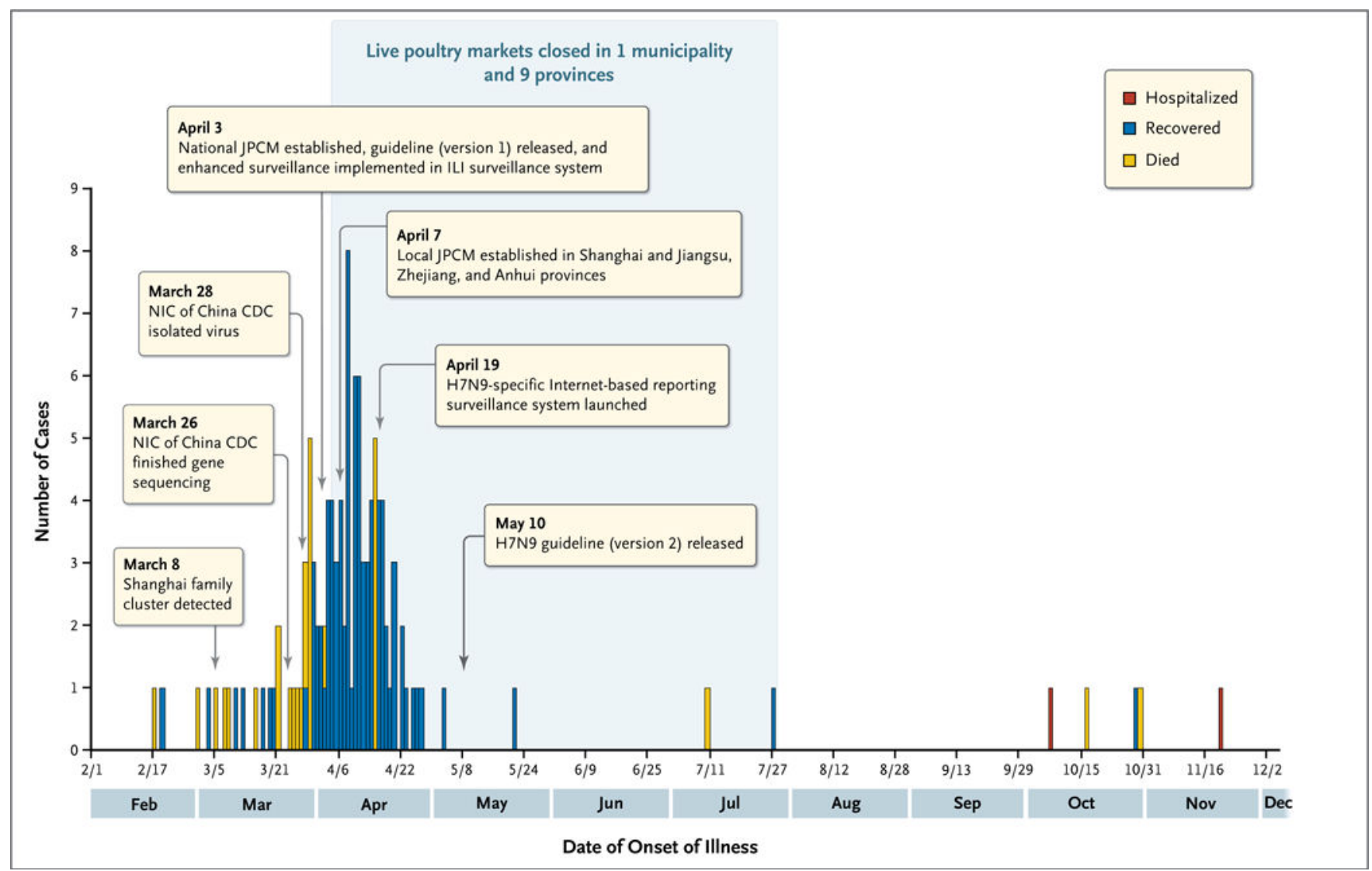

Figure 1. Date of Onset of Illness in 139 Patients with Confirmed H7N9 Virus Infection in China. Detailed information on closures of live poultry markets and emergency responses in 10 provinces and 2 municipalities is provided in Figure S2 in the Supplementary Appendix. CDC denotes Center for Disease Control and Prevention, ILI influenza-like illness, JPCM Joint Prevention and Control Mechanism, and NIC National Influenza Center. 


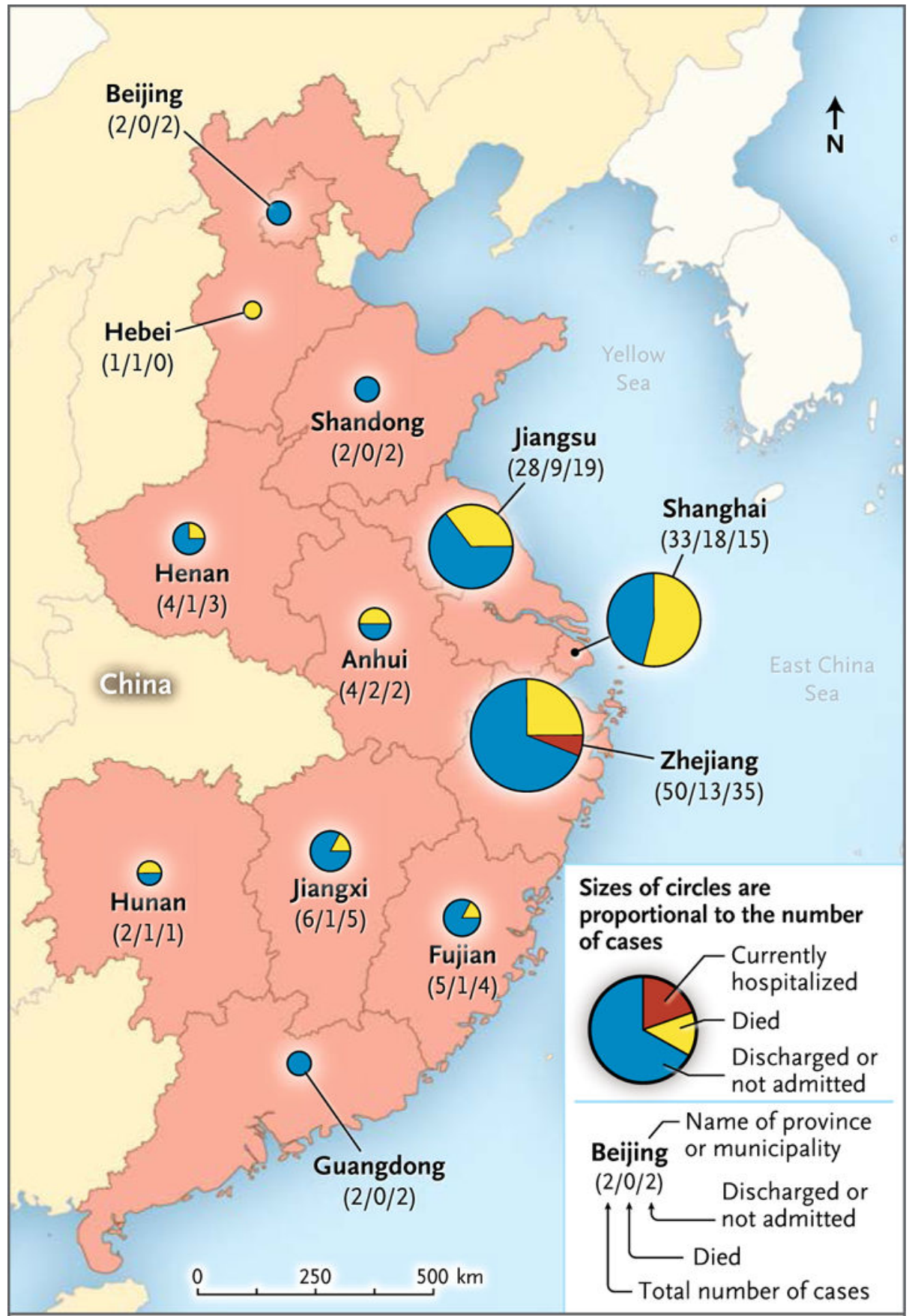

Figure 2.

Geographic Distribution of 139 Confirmed Cases of H7N9 Virus Infection in China, as of December 1, 2013. 


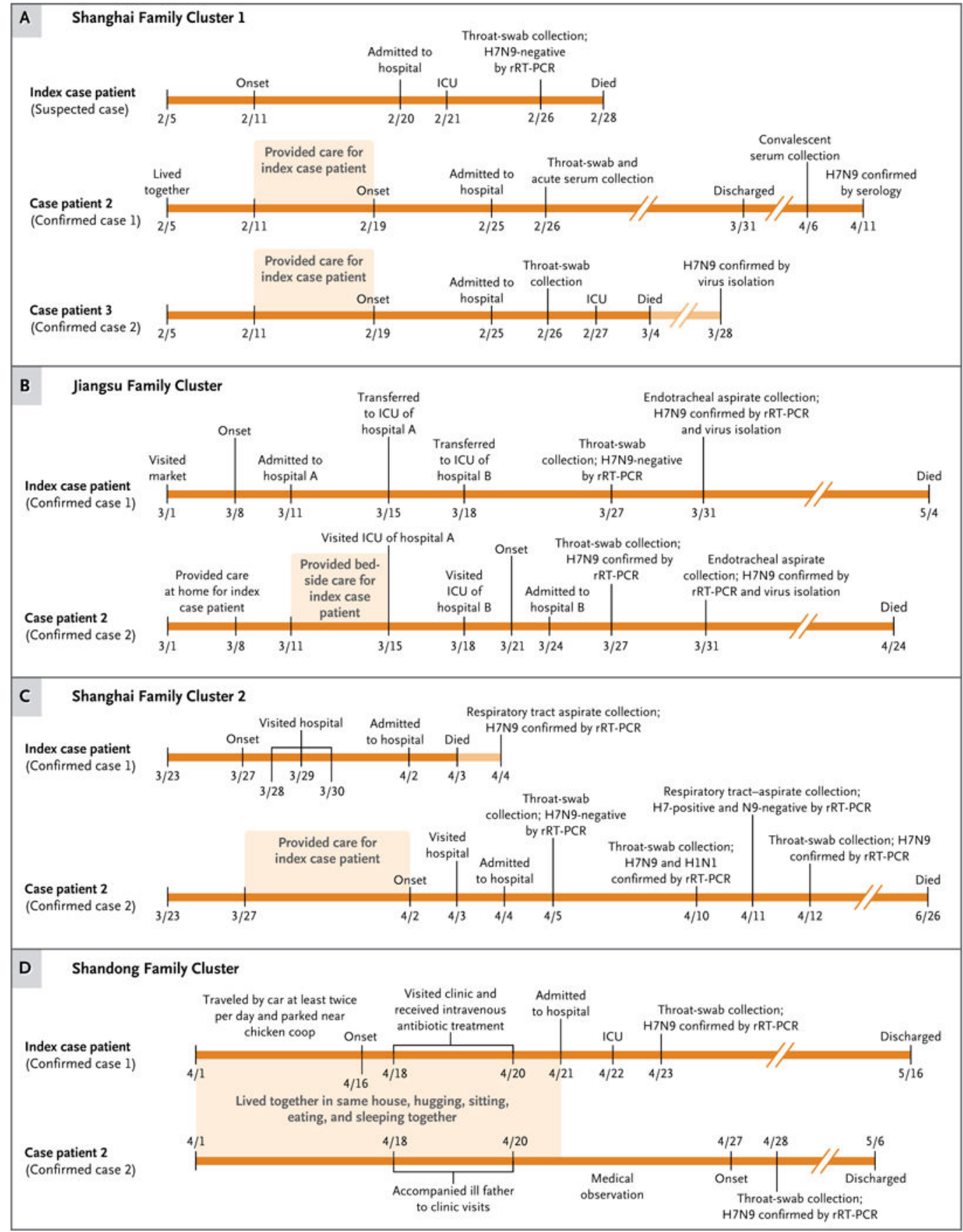

Figure 3. Timeline of Pertinent Exposures and Dates of Illness Onset in Four Family Clusters of Cases of H7N9 Virus Infection in China.

ICU denotes intensive care unit, and rRT-PCR real-time reverse-transcriptase-polymerasechain-reaction assay. 

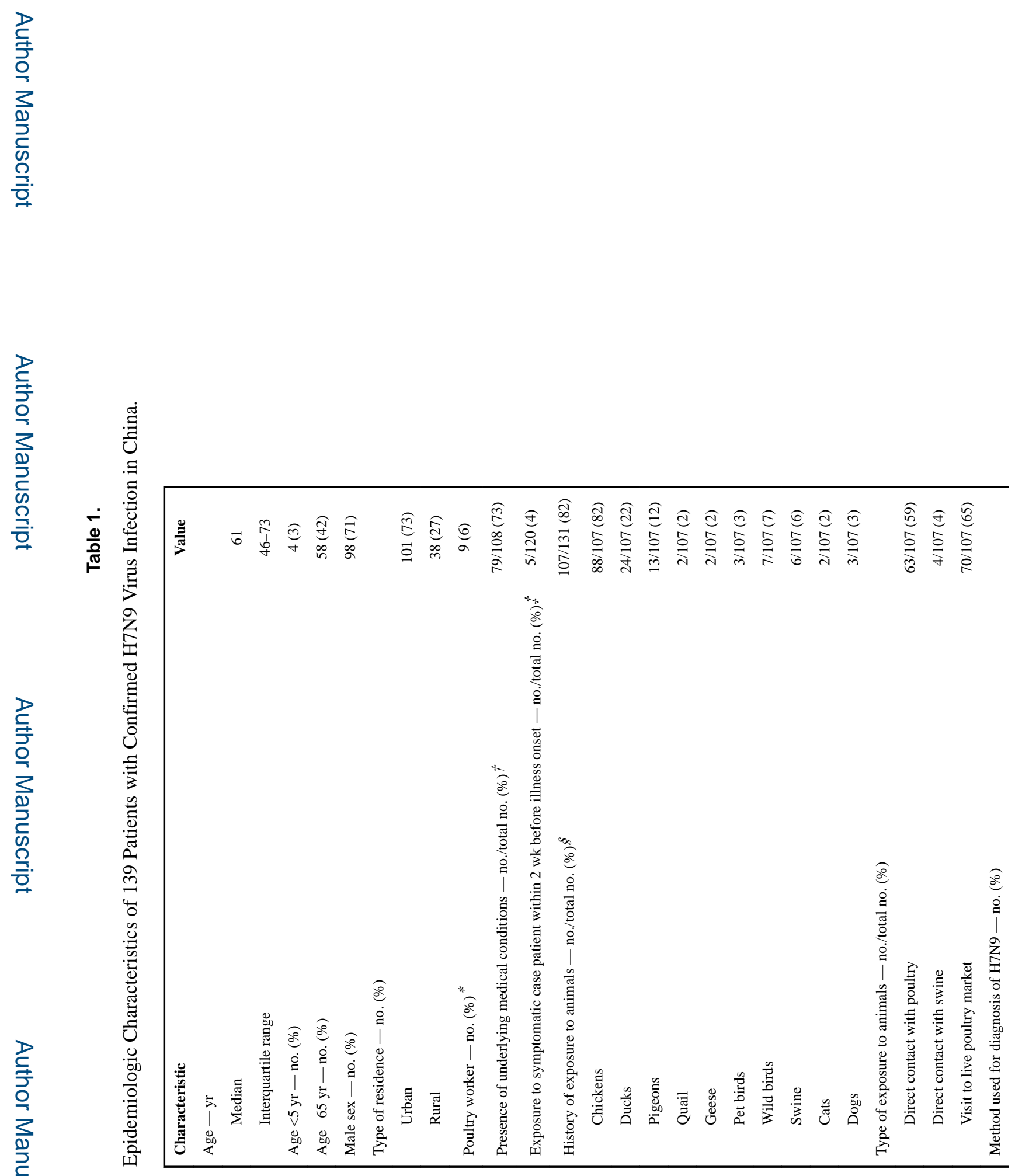

N Engl J Med. Author manuscript; available in PMC 2019 July 24. 


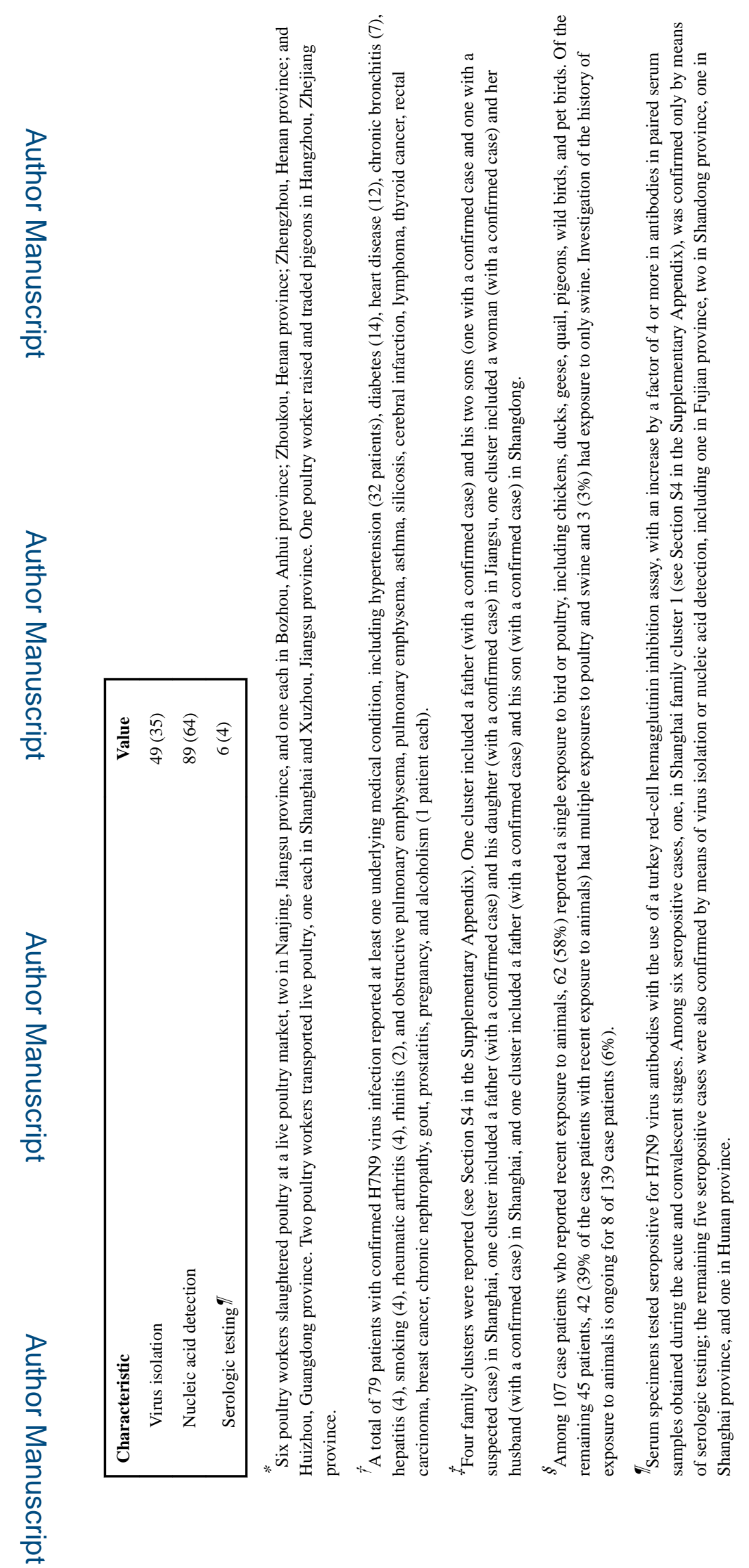

N Engl J Med. Author manuscript; available in PMC 2019 July 24. 
Table 2.

Clinical Characteristics and Medical Care Timelines for 139 Patients with Confirmed H7N9 Virus Infection in China. ${ }^{*}$

\begin{tabular}{|c|c|}
\hline Variable & $\begin{array}{l}\text { Patients with Confirmed } \\
\text { Cases }(\mathbf{N}=139)\end{array}$ \\
\hline \multicolumn{2}{|l|}{ Clinical outcome - no./total no. $(\%)^{\dagger}$} \\
\hline Required hospitalization & $137 / 139(99)$ \\
\hline Currently hospitalized & $2 / 139(1)$ \\
\hline Admitted to ICU & $65 / 103(63)$ \\
\hline Required mechanical ventilation & $56 / 91(62)$ \\
\hline Had ARDS & $48 / 83(58)$ \\
\hline Died in hospital ${ }^{\hbar}$ & $47 / 139(34)$ \\
\hline Recovered & $90 / 139(65)$ \\
\hline Oseltamivir treatment - no./total no. $(\%)$ & $79 / 109(72)$ \\
\hline \multicolumn{2}{|c|}{ Time from illness onset to oseltamivir treatment — days } \\
\hline Median & 6.0 \\
\hline Interquartile range & $5.0-9.0$ \\
\hline \multicolumn{2}{|c|}{ Time from illness onset to first medical care - days } \\
\hline Median & 1.0 \\
\hline Interquartile range & $0-3.0$ \\
\hline \multicolumn{2}{|c|}{ Time from illness onset to hospitalization — days } \\
\hline Median & 4.0 \\
\hline Interquartile range & $3.0-6.0$ \\
\hline \multicolumn{2}{|c|}{ Time from illness onset to ICU admission — days } \\
\hline Median & 7.0 \\
\hline Interquartile range & $5.0-9.0$ \\
\hline \multicolumn{2}{|c|}{ Time from illness onset to development of ARDS — days } \\
\hline Median & 7.0 \\
\hline Interquartile range & $5.0-9.0$ \\
\hline \multicolumn{2}{|l|}{ Time from illness onset to death — days } \\
\hline Median & 21.0 \\
\hline Interquartile range & $12.5-36.0$ \\
\hline
\end{tabular}

In the case of some characteristics, complete data were not available for all 139 patients with confirmed H7N9 virus infection. Case investigations are ongoing. ARDS denotes acute respiratory distress syndrome, and ICU intensive care unit.

${ }^{\dagger}$ A total of 87 patients with confirmed cases had been discharged as of December 1, 2013. One 2-year-old boy with a confirmed case in Shanghai was not hospitalized, because his illness was mild; he recovered 5 days after the onset of illness. A 25 -year-old woman with a confirmed case in Jiangsu province was not hospitalized, because her illness was mild, and she recovered 18 days after the onset of illness.

${ }^{*}$ One additional death occurred after hospital discharge and is not included as a death in the hospital. This was a case patient from Jiangsu province who recovered and was discharged from the hospital in August but died at home in September from an underlying medical condition. 